\title{
Gate-dependent orbital magnetic moments in carbon nanotubes
}

Jespersen, Thomas Sand; Grove-Rasmussen, Kasper; Flensberg, Karsten; Paaske, Jens; Muraki, K.; Fujisawa, T.; Nygård, Jesper

Published in:

Physical Review Letters

DOI:

10.1103/PhysRevLett.107.186802

Publication date:

2011

Document version

Early version, also known as pre-print

Citation for published version (APA):

Jespersen, T. S., Grove-Rasmussen, K., Flensberg, K., Paaske, J., Muraki, K., Fujisawa, T., \& Nygård, J. (2011). Gate-dependent orbital magnetic moments in carbon nanotubes. Physical Review Letters, 107(18), 186802. https://doi.org/10.1103/PhysRevLett.107.186802 


\title{
Gate-dependent spin-orbit coupling in multielectron carbon nanotubes
}

\author{
T. S. Jespersen ${ }^{1 \star \dagger}$, K. Grove-Rasmussen ${ }^{1,2 \star \dagger}$, J. Paaske ${ }^{1}$, K. Muraki $^{2}$, T. Fujisawa ${ }^{3}$, J. Nygård ${ }^{1}$ \\ and K. Flensberg ${ }^{1}$
}

\begin{abstract}
Understanding how the orbital motion of electrons is coupled to the spin degree of freedom in nanoscale systems is central for applications in spin-based electronics and quantum computation. Here we demonstrate such spin-orbit coupling in a carbon-nanotube quantum dot in the general multielectron regime and in the presence of finite disorder. Also, we find a systematic dependence of the spin-orbit coupling on the electron occupation of the quantum dot. Such a dependence has not been seen in any other system and follows from the curvature-induced spin-orbit-split Dirac spectrum of the underlying graphene lattice. Our findings suggest that the spin-orbit coupling is a general property of carbon-nanotube quantum dots, which should provide a unique platform for the study of spin-orbit effects and their applications.
\end{abstract}

T he interaction of the spin of electrons with their orbital motion has become a focus of attention in quantum-dot research. On the one hand, this spin-orbit interaction (SOI) provides a route for spin decoherence, which is unwanted for purposes of quantum computation ${ }^{1-3}$. On the other hand, if properly controlled, the SOI can be used as a means of electrically manipulating the spin degree of freedom ${ }^{4-7}$.

In this context, carbon nanotubes (CNTs) provide a number of attractive features, including large confinement energies and nearly nuclear-spin-free environment; most importantly, the details of the energy-level structure are theoretically well understood and modelled, as well as being experimentally highly reproducible. Remarkably, the SOI in nanotubes was largely overlooked in the first two decades of nanotube research and was only recently demonstrated by Kuemmeth et al. for the special case of one and two carriers in ultraclean CNT quantum dots ${ }^{3,8,9}$. Except for these reports, the SOI in nanotubes is experimentally unexplored. Theoretically, the focus has exclusively been on the SOI-modified band structure of disorder-free nanotubes ${ }^{10-14}$. Therefore, two important questions remain: how the effective SOI depends on electron filling and how it appears in the general case of quantum dots subject to disorder. Here we answer these two questions.

First, by low-temperature electron transport we demonstrate the presence of a significant SOI in a disordered CNT quantum dot holding hundreds of electrons. We identify and analyse the role of SOI in the energy spectrum for one, two and three electrons in the fourfold degenerate CNT electronic shells, thus describing shells at any electron filling. By rotating the sample, we present spectroscopy of the same charge states for magnetic fields both parallel and perpendicular to the nanotube axis, thus controlling the coupling to the orbital magnetic moment. Remarkably, a single-electron model taking into account both SOI and disorder quantitatively describes all essential details of the multielectron quantum-dot spectra. Second, by changing the electron occupancy we are able to tune the effective SOI in accordance with the expected curvature-induced spin-orbit splitting of the underlying graphene Dirac spectrum ${ }^{1,11-14}$. Such systematic dependence has not been demonstrated in any other material system and may enable a new range of spin-orbit-related applications. This microscopic understanding and detailed modelling is in stark contrast to situations encountered in alternative strong-SOI quantum-dot materials, such as InAs or InSb nanowires, where the effective SOI arises from bulk crystal effects combined with unknown contributions from surface effects, strain and crystal defects ${ }^{15}$. These systems often exhibit semi-random fluctuations of, for example, the $g$-factor as single electrons are added ${ }^{16,17}$. Thus, beyond fundamental interest and the prospect of realizing recent proposals of SOI-induced spin control in CNTs (refs 1,18), our findings pave the way for new designs of experiments using $\mathrm{SOI}$ in quantum dots.

\section{Breaking the fourfold degeneracy}

Our experimental set-up is presented in Fig. 1a. We fabricate devices of single-wall CNTs on highly doped Si substrates capped with an insulating layer of $\mathrm{SiO}_{2}$ (see the Methods section). The size of the quantum dot is defined by the contact separation $(400 \mathrm{~nm})$ and the electrical properties are investigated in a voltage-biased two-terminal configuration, applying a voltage $V_{\text {sd }}$ between source and drain contacts and measuring the resulting current $I$. The differential conductance $\mathrm{d} I / \mathrm{d} V_{\text {sd }}$ is measured by standard lock-in techniques. When biased with a voltage $V_{g}$, the Si substrate acts as an electrostatic gate controlling the electron occupancy of the dot. The device is measured at temperature $T=100 \mathrm{mK}$ in a ${ }^{3} \mathrm{He} /{ }^{4} \mathrm{He}$ dilution refrigerator, fitted with a piezo-rotator enabling in-plane rotations of the sample in magnetic fields up to $9 \mathrm{~T}$.

Figure 1b shows a typical measurement of $\mathrm{d} I / \mathrm{d} V_{\text {sd }}$ versus $V_{\text {sd }}$ and $V_{g}$ in the multielectron regime of a small-bandgap semiconducting nanotube. The pattern of diamond-shaped regions of low conductance is expected for a quantum dot in the Coulomb blockade regime, and within each diamond the quantum dot hosts a fixed number of electrons $N$, increasing one by one with increasing $V_{g}$. The energy $E_{\text {add }}$ required for adding a single electron is seen as the diamond heights and has been extracted in Fig. 1c. The

\footnotetext{
${ }^{1}$ Niels Bohr Institute \& Nano-Science Center, University of Copenhagen, Universitetsparken 5, DK-2100 Copenhagen, Denmark, ${ }^{2}$ NTT Basic Research Laboratories, NTT Corporation, 3-1 Morinosato-Wakamiya, Atsugi 243-0198, Japan, ${ }^{3}$ Research Center for Low Temperature Physics, Tokyo Institute of Technology, Ookayama, Meguro, Tokyo 152-8551, Japan. †These authors contributed equally to this work. *e-mail: tsand@fys.ku.dk; k_grove@fys.ku.dk.
} 


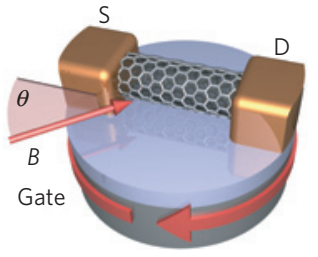

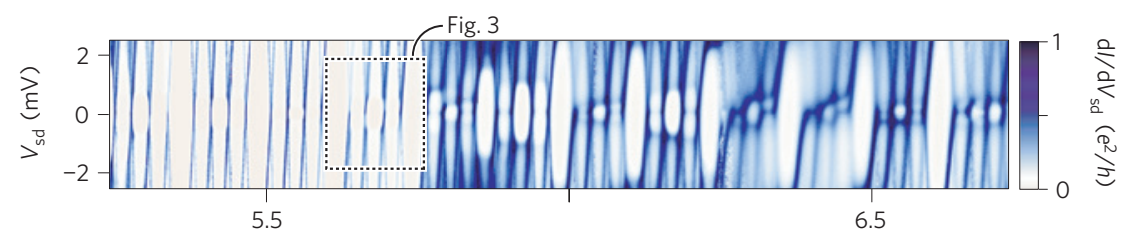

$V_{g}(V)$

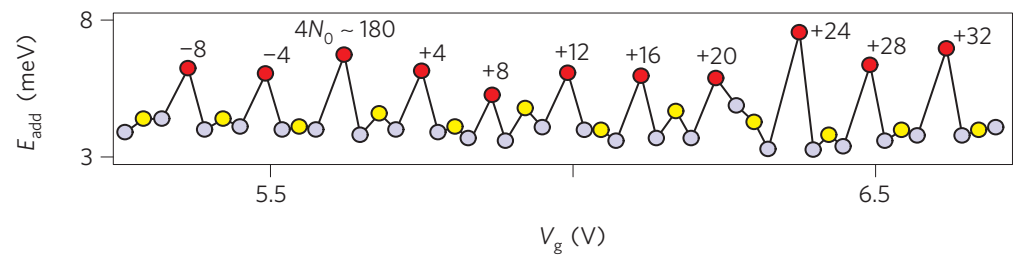

Figure 1 | Fourfold periodic nanotube spectrum. a, Schematic illustration of the device and set-up. CNT quantum dots are measured at $T=100$ mK in a standard two-terminal configuration in a cryostat modified to enable measurements in a high magnetic field at arbitrary in-plane angles $\theta$ to the CNT axis. b, Typical measurement of the differential conductance $d / / d V_{\text {sd }}$ versus source-drain bias $V_{\text {sd }}$ and gate voltage $V_{g}$ for a multielectron $C N T$ quantum dot. c, Addition energy as a function of $V_{g}$. In $\mathbf{b}$ and $\mathbf{c}$ the characteristic filling of four-electron shells is clearly seen.

four-electron periodicity clearly observed in Fig. 1b,c reflects the near fourfold degeneracy in the nanotube energy spectrum ${ }^{19,20}$; one factor of two from the intrinsic spin $(\uparrow, \downarrow)$ and one factor of two from the so-called isospin $\left(K, K^{\prime}\right)$ that stems from the rotational symmetry of the nanotube-electrons orbit the CNT in a clockwise or anticlockwise direction. As is generally observed ${ }^{19-23}$, the addition energy for the second electron in each quartet (yellow in Fig. 1c) exceeds those for one and three. This was previously interpreted as a result of disorder-induced coupling $\Delta_{\mathrm{KK}^{\prime}}$ of the clockwise and anticlockwise states ${ }^{21,24}$ that splits the spectrum into two spin-degenerate pairs of bonding/antibonding states separated by $\Delta_{\mathrm{KK}^{\prime}}$. As mentioned, Kuemmeth et al. recently showed that for the first electron in an ultraclean suspended nanotube quantum dot the splitting was instead dominated by the spin-orbit coupling. The first question we address here is whether SOI also appears in the many-electron regime and how it may be modified or masked by disorder.

\section{Modelling spin-orbit coupling and disorder}

Carrying out level spectroscopy with a magnetic field $B$ applied either parallel $\left(B_{\|}\right)$or perpendicular $\left(B_{\perp}\right)$ to the nanotube axis proves to be a powerful tool to analyse the separate contributions from disorder and spin-orbit coupling. This is illustrated in Fig. 2a$d$, which shows calculated single-particle energy-level spectra for four limiting combinations of $\Delta_{\mathrm{KK}^{\prime}}$ and the effective spinorbit coupling $\Delta_{\text {SO }}$ (all limits are relevant for nanotube devices depending on the degree of disorder and CNT structure ${ }^{13,14}$; details of the model are provided in Supplementary Information). In all cases a parallel field separates the four states into pairs of increasing (K-like states) and decreasing ( $\mathrm{K}^{\prime}$-like states) energies. The magnitude of the shift is given by the orbital $g$-factor $g_{\text {orb }}$ reflecting the coupling of $B_{\|}$to the orbital magnetic moment caused by motion around the CNT (ref. 25). Further, each pair exhibits a smaller internal splitting owing to the Zeeman effect. Figure $2 \mathrm{~b}$ shows the disorder-induced coupling of $\mathrm{K}$ and $\mathrm{K}^{\prime}$ states resulting in an avoided crossing at $B_{\|}=0$ and the zero-field splitting discussed above. In the opposite limit with SOI only (Fig. 2c), the zero-field spectrum is also split into two doublets, but the field dependence is markedly different and no avoided crossing appears. In the simplest picture, this behaviour originates from coupling of the electron spin to an effective magnetic field $\mathbf{B}_{\mathrm{SO}}=-(\mathbf{v} \times \mathbf{E}) / c^{2}$ experienced by the electron as it moves with velocity $\mathbf{v}$ in an electric field $\mathbf{E}$. Here the speed of light, $c$, reflects the relativistic origin of the effect. In nanotubes, the curvature of the graphene lattice generates an effective radial electric field, and as the
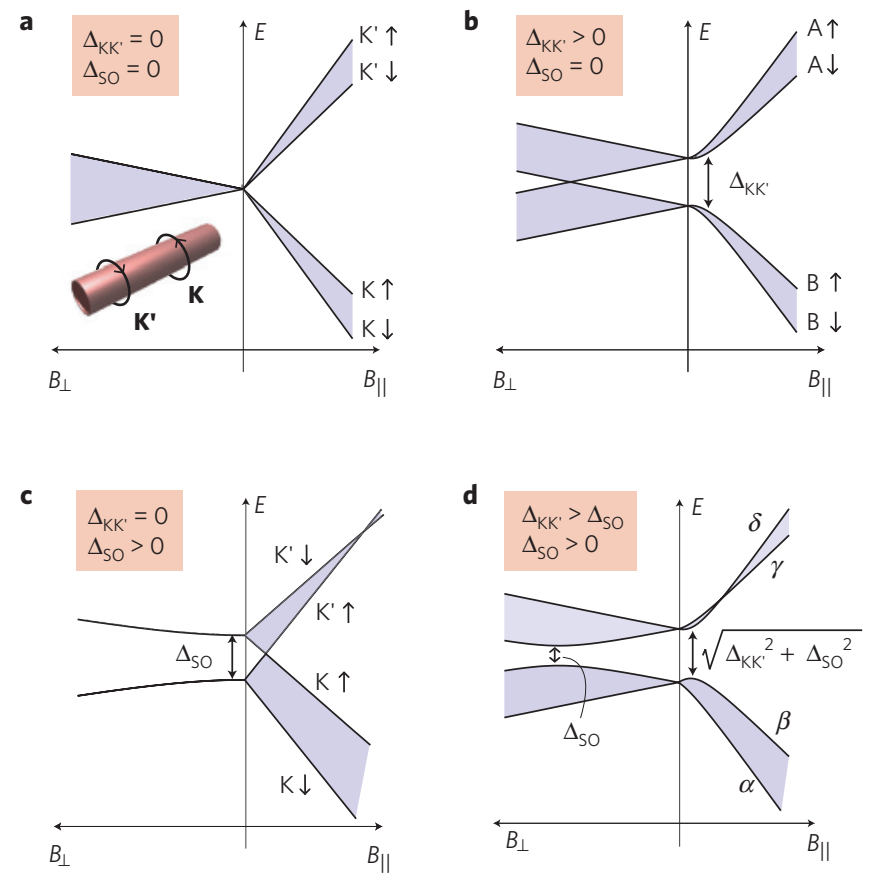

Figure 2 | Role of spin-orbit interaction and disorder for the nanotube energy spectrum. a-c, Calculated single-particle energy spectrum for a quartet in the conduction band as a function of magnetic field applied perpendicular $\left(B_{\perp}\right)$ and parallel $\left(B_{\|}\right)$to the CNT axis in the limiting cases of neither SOI nor disorder (a), disorder alone (b), SOI alone (c), and the two combined $\Delta_{\mathrm{KK}^{\prime}}>\Delta_{\mathrm{SO}}>0$. d, Depending on the CNT type, electron filling and degree of disorder, all four situations can occur.

velocity is mainly circumferential (and opposite for $\mathrm{K}$ and $\mathrm{K}^{\prime}$ ) $\mathbf{B}_{\mathrm{SO}}$ polarizes the spins along the nanotube axis and favours parallel or antiparallel alignment of the spin and orbital magnetic momentum depending on the sign of $\Delta_{\mathrm{SO}}$. Thus, even in the absence of disorder, the spectrum splits into two Kramers doublets $\left(K \downarrow, K^{\prime} \uparrow\right)$ and $\left(\mathrm{K} \uparrow, \mathrm{K}^{\prime} \downarrow\right)$ separated by $\Delta_{\mathrm{SO}}$. Interestingly, as a perpendicular field does not couple $\mathrm{K}$ and $\mathrm{K}^{\prime}$ the doublets do not split along $B_{\perp}$ (Fig. $2 \mathrm{c}$ ). As a consequence, the $g$-factor, when measured in a perpendicular magnetic field, will vary from zero when $\Delta_{\mathrm{SO}} \gg \Delta_{\mathrm{KK}}$ (Fig. 2c) to two in the opposite limit (Fig. 2b; ref. 26).

The final case, including both disorder and SOI, is of particular importance for the present study, and the calculated spectrum 


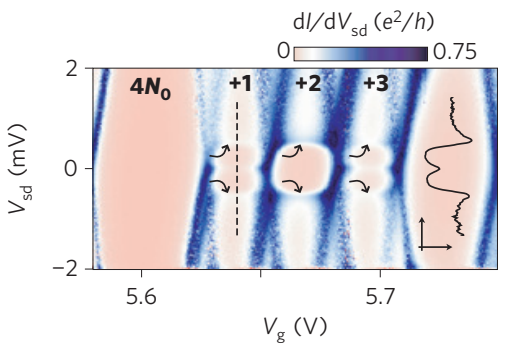

d
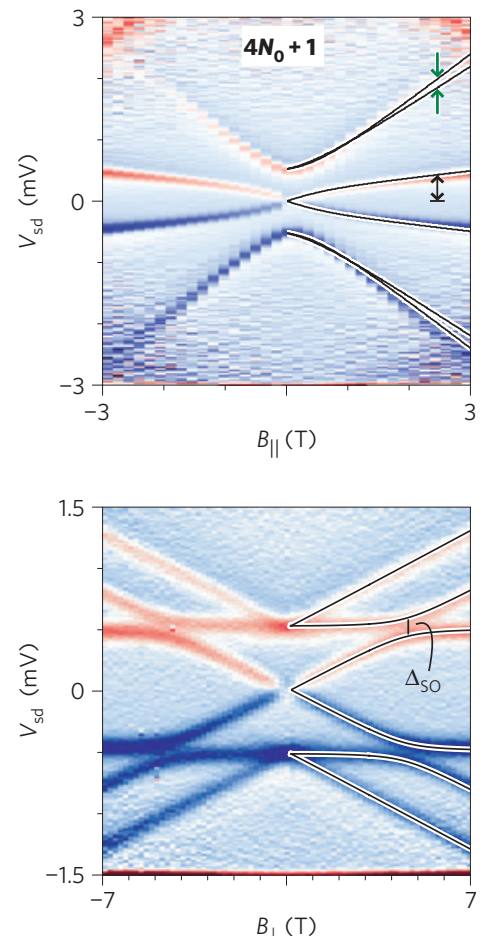

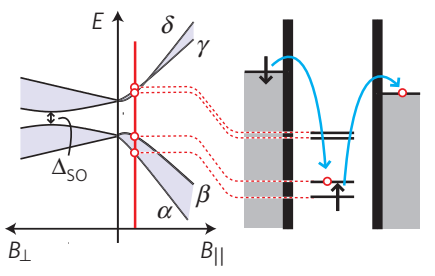

e

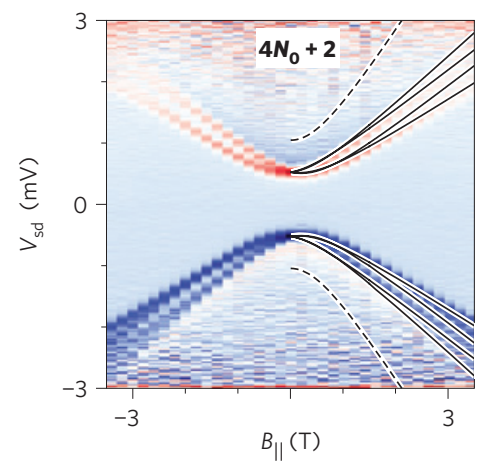

h

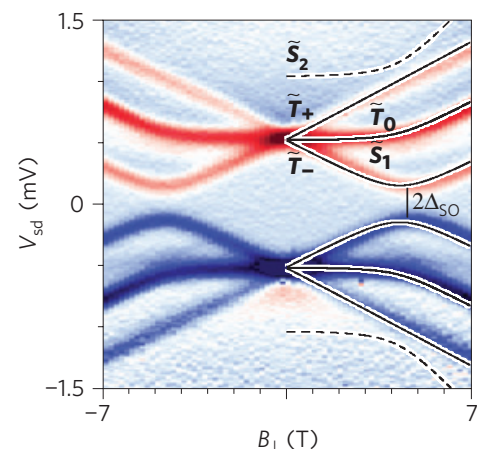

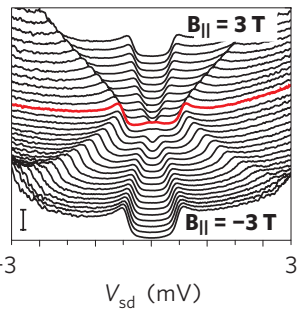

f

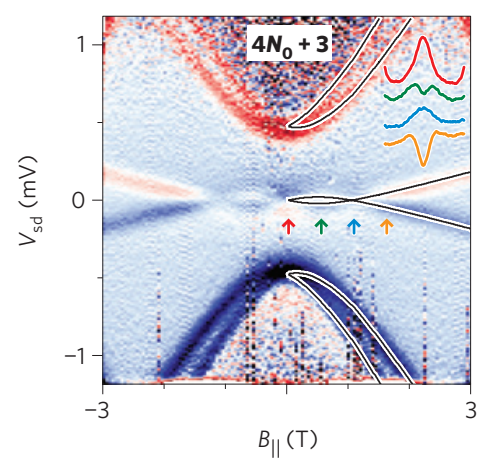

i

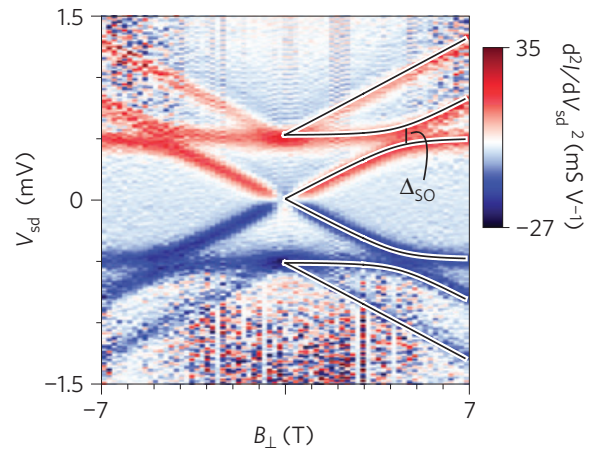

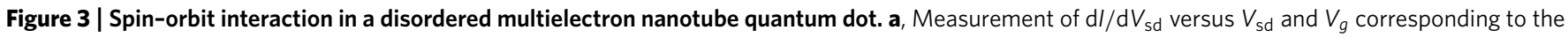
consecutive addition of four electrons to an empty shell (indicated in Fig. 1b). A strong tunnel coupling results in significant cotunnelling, which is evident as horizontal lines truncating the diamonds (arrows). The black trace shows a cut along the dashed line. $\mathbf{b}$, Schematic illustration of the relevant inelastic

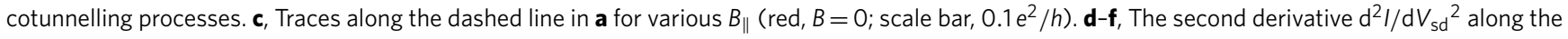
centre of the $N_{0}+1, N_{0}+2$ and $N_{0}+3$ diamond, respectively, as a function of a parallel magnetic field. Peaks/dips appear at inflection points of the differential conductance and thus correspond to the energy difference between ground and excited states. In $\mathbf{f}$ the inset shows $\mathrm{d} / / \mathrm{d} V_{\mathrm{sd}}$ versus $-0.3<V_{\text {sd }}<0.3 \mathrm{mV}$ and $B_{\|}=0 ; 0.55 ; 1.1 ; 1.65 \mathrm{~T}$ (arrows) illustrating the splitting and SOl-induced reappearance of a zero-bias Kondo resonance. $\mathbf{g}-\mathbf{i}$, As $\mathbf{d}-\mathbf{f}$ but measured as a function of $B_{\perp}$. The effective spin-orbit coupling appears directly as the avoided crossings indicated by $\Delta$ so. In $\mathbf{d}$-i the black lines result from the single-particle model with parameters $\Delta_{\mathrm{SO}}=0.15 \mathrm{meV}, \Delta_{\mathrm{KK}^{\prime}}=0.45 \mathrm{meV}$ and $g_{\text {orb }}=5.7$. The dashed lines in $\mathbf{e}, \mathbf{h}$ correspond to excitations to the two-electron singlet-like $\tilde{S}_{2}$ state, which cannot be reached by promoting a single electron from the ground state ( $\tilde{S}_{0}$ ) and is therefore expected to be absent from the measurement.

is shown in Fig. $2 \mathrm{~d}$ for $\Delta_{\mathrm{KK}^{\prime}}>\Delta_{\mathrm{SO}}$. Importantly, the effects of SOI are not masked despite the dominating disorder: for parallel field, SOI remains responsible for an asymmetric splitting of the Kramers doublets $(\alpha, \beta)$ versus $(\delta, \gamma)$, and the appearance of an extra degeneracy in the spectrum at finite field ( $\delta$ and $\gamma$ states). In a perpendicular field, the effect of SOI is to suppress the Zeeman splitting of the two doublets, and as the eigenstates of the SOI have spins along the nanotube axis it couples the states with spins polarized along $B_{\perp}$, resulting in the avoided crossing indicated in the figure. Note that for the present study the weak perpendicular electric field from the back-gate can be disregarded as a source of SOI as it couples different longitudinal modes, that is, states belonging to different electronic shells.

\section{Spin-orbit interaction revealed by spectroscopy}

With Fig. 2 in mind, we now focus on the quartet with $4 N_{0} \approx 180$ electrons highlighted in Fig. $1 \mathrm{~b}$ and expanded in
Fig. 3a. To investigate the level structure we carry out cotunnelling spectroscopy, as illustrated in the schematic Fig. 3b (ref. 27): in Coulomb blockade, whenever $e\left|V_{\text {sd }}\right|$ matches the energy of a transition from the ground state $\alpha$ to an excited state $(\beta, \gamma, \delta)$, inelastic cotunnel processes, which leave the quantum dot in the excited state, become available for transport. This significantly increases the current and gives rise to steps in the conductance. These appear as gate-independent features in Fig. 3a (arrows) and are clearly seen in the inset, showing a trace through the centre of the one-electron $\left(4 N_{0}+1\right)$ diamond along the dashed line. Thus following the magnetic-field dependence of this trace, as shown in Fig. 3c, maps out the level structure. The energies of the excitations are given by the inflection points of the curve (that is peaks/dips of $\mathrm{d}^{2} I / \mathrm{d} V_{\mathrm{sd}}{ }^{2}$; ref. 28) and the level evolution is therefore directly evident in Fig. $3 \mathrm{~d}-\mathrm{i}$, which shows colour maps of the second derivative versus $V_{\text {sd }}$ and $B_{\perp}, B_{\|}$for $V_{g}$ positioned in the centre of the one-, two- and three-electron charge 


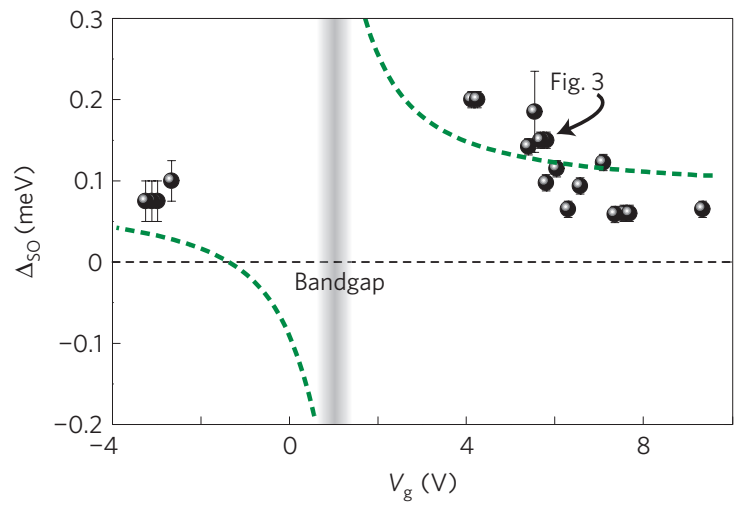

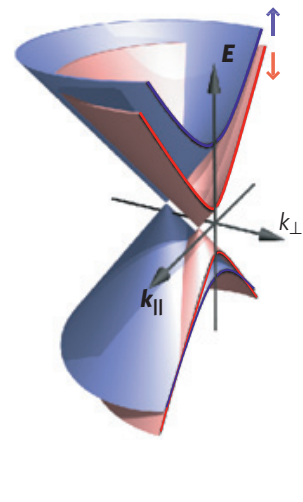

c

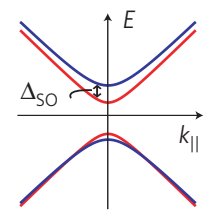

d

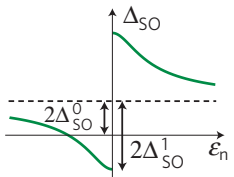

Figure 4 | Tuning $\Delta_{\text {so }}$ in accordance with the curvature-induced spin-orbit splitting of the nanotube Dirac spectrum. a, Measured effective spin-orbit coupling strength as a function of $V_{g}$ extracted from spectroscopy measurements like those in Fig. 3, repeated for multiple shells. The investigated valence-band states correspond to shells with $116,128,132$ and 136 holes. The dashed line is a fit to the theory. When $\Delta_{\text {so }}$ is determined by avoided crossings, the uncertainty is smaller than the size of the point. Data points with error bars correspond to cases where no clear avoided crossings could be identified owing to either tunnel-coupling-induced peak broadening (valence-band data) or insufficient range of $B$. $\Delta$ so values are then determined by fitting the entire calculated level structure to the measurement and error bars illustrate the range of values for which good correspondence is obtained. b, Graphene dispersion cones around one K point of the graphene Brillouin zone. Owing to SOI the spin-up (blue) and spin-down (red) Dirac cones are split in both the vertical $(E)$ and $k_{\perp}$ directions. The cut shows the resulting CNT band structure for a small-bandgap nanotube also shown in c. d, Expected dependence of $\Delta_{\mathrm{SO}}$ on $\varepsilon_{N}$ highlighting the two SOI contributions $\Delta_{\mathrm{SO}}^{0}$ and $\Delta_{\mathrm{SO}}^{1}$

states. As explained below, the SOI is clearly expressed in all three spectra.

Consider first the one-electron case: in a parallel field (Fig. 3d) the asymmetric splitting of the two doublets is evident (black versus green arrows), and applying the field perpendicularly (Fig. $3 \mathrm{~g}$ ) the SOI is directly expressed as the avoided crossing indicated in the figure. The measurement is in near-perfect agreement with the single-particle excitation spectrum calculated by subtracting the energies of Fig. $3 \mathrm{~b}$ and shown by the solid lines. The calculation depends on only three parameters: $\Delta_{\mathrm{SO}}=0.15 \mathrm{meV}$ set directly by the avoided crossing, $\Delta_{\mathrm{KK}^{\prime}}=0.45 \mathrm{meV}$ determined from the zero-field splitting of the doublets (see Fig. $2 \mathrm{~d}$ ), and $g_{\text {orb }}=5.7$ set by the slopes of the excitation lines from $\alpha$ to $\gamma, \delta$ in $\mathrm{d}$.

Consider now the role of SOI for the doubly occupied CNT quartet. This situation is of particular importance for quantum computation as a paradigm for preparation of entangled states and a fundamental part of Pauli blockade in double quantum $\operatorname{dots}^{29}$. Figure $3 \mathrm{e}, \mathrm{h}$ shows the measured spectra in parallel and perpendicular fields respectively. The model perfectly describes the measurement and now contains no free parameters, as these are fixed by the one-electron measurement. Six states are expected: the singlet-like ground state $\tilde{S}_{0}$ formed by the two electrons occupying the low-energy Kramers doublet, three triplet-like states $\tilde{T}_{-}, \tilde{T}_{0}, \tilde{T}_{+}$and a singlet-like state $\tilde{S}_{1}$, which all use one state from each doublet, and the singlet-like $\tilde{S}_{2}$ with both electrons occupying the high-energy doublet-these states are not the conventional spin singlets and triplets as they are modified by SOI. The ground state $\tilde{S}_{0}$ does not appear directly in the measurement, but sets the origin for the cotunnelling excitations. The excitation to the high-energy $\tilde{S}_{2}$ (dashed line) is absent from the experiment, as it cannot be reached by promoting only a single electron from $\tilde{S}_{0}$. In Fig. $3 \mathrm{~h}$ excitations to $\tilde{T}_{-}$and $\tilde{T}_{+}$are clearly observed, whereas excitations to $\tilde{S}_{1}$ and $\tilde{T}_{0}$ merge into a single high-intensity peak, showing that any exchange splitting $J$ is below the spectroscopic line width $\approx 100 \mu \mathrm{eV}$ (ref. 23 ). In other quartets an exchange splitting is indeed observed (see Supplementary Information). In the two-electron spectra the SOI is directly expressed as the avoided crossing at $B_{\perp} \approx 4.5 \mathrm{~T}$ accompanying the $\tilde{S}_{0} \leftrightarrow \tilde{T}_{\text {- }}$ ground-state transition ${ }^{15}$. In quartets of yet stronger tunnel coupling it is replaced by a singlet-triplet Kondo resonance ${ }^{30}$.
Finally, the spectrum of three electrons in the four-electron shell is equivalent to that of a single hole in a full shell; at low fields the $\delta$-state becomes the ground state and $\gamma$ the first excited state, whereas $\alpha$ and $\beta$ then constitute the excited doublet. As seen by comparing Fig. 2b,d SOI breaks the intra-shell electronhole symmetry of the nanotube spectrum. This is evident in the experiment when comparing Fig. 3d,f: in 3f, increasing $B_{\|}$, the lowest excited state $\gamma$ barely separates from the ground state $\delta$ and at $B_{\|}=1.1 \mathrm{~T}$ they cross again, causing a ground-state transition. At the crossing point, the spin-degenerate ground state results in a zero-bias Kondo peak (see inset) ${ }^{31}$. Interestingly, this degeneracy also forms the qubit proposed in ref. 1 . For the $B_{\perp}$ dependence the one- and three-electron cases remain identical and Fig. 3 i exhibits again the SOI-induced avoided crossings.

\section{Gate-dependent spin-orbit coupling}

Having established the presence of SOI in the general many-electron disordered quantum dot, we now focus on the dependence of $\Delta_{\mathrm{SO}}$ on the quantum-dot occupation. To this end, we have repeated the spectroscopy of Fig. 3 for a large number of CNT quartets and in each case extracted $\Delta_{\text {SO }}$ by fitting to the single-particle model (all underlying data are presented in Supplementary Information). Interestingly, quartets in the valence band have the one- and threeelectron spectra qualitatively reversed compared with Fig. 3; thus, although SOI favours parallel spin and orbital magnetic moments in the conduction band, it favours antiparallel polarization for electron states in the valence band. Thus in this sense electron-hole symmetry is preserved, corresponding to positive $\Delta_{\mathrm{SO}}$ for both the valence and conduction bands. This contrasts the situation of Kuemmeth et al. ${ }^{8}$ and is surprising, as electron-hole symmetry is broken in the conventional picture of SOI as acting equivalently to an effective parallel magnetic field. Figure $4 \mathrm{a}$ shows the resulting gate dependence of $\Delta_{\text {So }}$. A gradual decrease is observed as electrons are added to the conduction band and the values exceed those of the valence band at the same electron/hole filling. This agrees qualitatively with recent models of the curvature-induced spinorbit splitting of the underlying graphene lattice, as we now discuss.

The magnitude of $\Delta_{S O}$ is given by the spin-orbit splitting of the underlying graphene band structure. For flat graphene this splitting is very weak ( $\Delta_{\text {SO }}^{\text {graphene }} \sim 1 \mu \mathrm{eV}$; ref. 12 ), as it is second order in the already weak atomic SOI of carbon $\Delta_{\text {SO }}^{C} \sim 8 \mathrm{meV}$. In nanotubes, 
however, the curvature induces a coupling between the $\pi$ - and $\sigma$-bands and generates a curvature-induced spin-orbit splitting, which is first order in the atomic SOI and thus greatly enhances

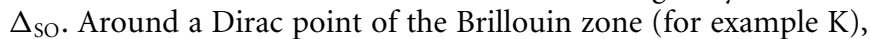
the graphene band structure appears as in Fig. 4b (refs 11-14): the spin-up and spin-down Dirac cones are split by SOI both in energy and along $k_{\perp}$, the momentum in the circumferential direction of the CNT. The schematic diagram in Fig. $4 \mathrm{c}$ also highlights the CNT band structure obtained by imposing periodic boundary conditions on $k_{\perp}$. In a finite-length CNT quantum dot the wavevector along the nanotube axis, $k_{\|}$, is also quantized, and letting $\varepsilon_{N}$ denote the energy of the $N$ th longitudinal mode the effective SOI for a small-bandgap CNT becomes

$$
\begin{aligned}
\Delta_{\mathrm{SO}} & =E_{\uparrow}^{\mathrm{K}}-E_{\downarrow}^{\mathrm{K}} \\
& =2\left(\Delta_{\mathrm{SO}}^{0} \mp \frac{\Delta_{\mathrm{SO}}^{1}}{\sqrt{1+\left(\varepsilon_{N} / \Delta_{g}\right)^{2}}}\right)
\end{aligned}
$$

Here the upper (lower) sign refers to the conduction (valence) band, $\Delta_{g}$ is the curvature-induced energy gap ${ }^{32,33}$ and the two terms $\Delta_{\text {SO }}^{0}$ and $\Delta_{\text {SO }}^{1}$ are the band-structure spin-orbit parameters due to curvature ${ }^{11-14}$. The separate contributions of the two terms are illustrated in Fig. 4 d. $\Delta_{\text {SO }}^{1}$ was found in the work of Ando ${ }^{10}$ and accounts for the $k_{\perp}$ separation of the Dirac cones in Fig. $4 \mathrm{~b}$. For the CNT band structure this term acts equivalently to an Aharonov-Bohm flux from a parallel spin-dependent magnetic field, which changes the quantization conditions in the $k_{\perp}$ direction. Characteristically, its contribution to $\Delta_{\mathrm{SO}}$ decreases with the number of electrons in the $\operatorname{dot}\left(\varepsilon_{N}\right)$ and reverses sign for the valence band. This contrasts the $\varepsilon_{N}$-independent contribution from the recently predicted $\Delta_{\mathrm{SO}}^{0}$ term ${ }^{12-14}$, which acts as an effective valleydependent Zeeman term and accounts for the energy splitting of the Dirac cones in Fig. $4 \mathrm{~b}$. For the nanotube studied in ref. 8 $\Delta_{\text {SO }}$ has opposite signs for electrons and holes, that is, $\left|\Delta_{\text {SO }}^{1}\right|>$ $\left|\Delta_{\text {SO }}^{0}\right|$. In our case, the positive values measured in the valence band demonstrate the opposite limit of dominating $\Delta_{\mathrm{SO}}^{0}$ due to the larger filling. The measured gate dependence of $\Delta_{\mathrm{SO}}$ thus qualitatively agrees with the spin-orbit splitting of the graphene Dirac spectrum caused by the curvature of the nanotube, and fitting to equation (1) (Fig. $4 \mathrm{a}$, dashed line) yields $\Delta_{\text {SO }}^{0}=40 \pm$ $15 \mu \mathrm{eV}$ and $\Delta_{\text {SO }}^{1}=-170 \pm 15 \mu \mathrm{eV}$. Band-structure models relate these parameters to the structure of the nanotube ${ }^{12-14}: \Delta_{\mathrm{SO}}^{0}=$ $\left(\alpha_{2} / \beta\right) \Delta_{\text {SO }}^{C} \Delta_{g} D$ and $\Delta_{\text {SO }}^{1}=-\alpha_{1} \Delta_{\text {SO }}^{C} / D$, where $D$ is the nanotube diameter and $\alpha_{1}, \alpha_{2}, \beta$ constants that depend on the CNT class (semiconducting, small bandgap). Typically CVD-grown singlewall CNTs have diameters in the range $1-3 \mathrm{~nm}$, whereas obtaining $D$ from the measured values of $g_{\text {orb }}$ gives $D \approx 10 \mathrm{~nm}$. Thus taking $D=1-10 \mathrm{~nm}$ we estimate $\left(\alpha_{2} / \beta\right)^{\exp }=(0.2-6) \times 10^{-4}(\mathrm{~nm} \mathrm{meV})^{-1}$ and $\alpha_{1}^{\exp }=(0.02-0.3) \mathrm{nm}$. Although $\alpha_{1}^{\exp }$ is consistent with the value quoted in the theoretical literature $(0.048 \mathrm{~nm}$; ref. 14$)$, the predicted $\left(\alpha_{2} / \beta\right)$ value $-2 \times 10^{-3}(\mathrm{~nm} \mathrm{meV})^{-1}$ does not match the experimental value or sign. This sign reflects whether the valence or conduction band experiences the higher spin-orbit splitting: for small-bandgap nanotubes theory predicts the valence-band spin-orbit splitting to exceed the conduction-band value $e^{14,34}$ inconsistent with the present measurement and ref. 8. The origin of this discrepancy remains unknown, and work on SOI in nanotubes with known chirality is needed to make further progress.

\section{Methods}

The devices are made on a highly doped silicon wafer terminated by $500 \mathrm{~nm}$ of $\mathrm{SiO}_{2}$. Alignment marks $(\mathrm{Cr}, 70 \mathrm{~nm})$ are defined by electron-beam lithography before deposition of catalyst islands made of iron nitrate $\left(\mathrm{Fe}\left(\mathrm{NO}_{3}\right)_{3}\right)$, molybdenum acetate and alumina support particles ${ }^{35}$. The sample is then transferred to a furnace, where single-wall carbon nanotubes are grown by chemical vapour deposition at $850-900^{\circ} \mathrm{C}$ in an atmosphere of hydrogen, argon and methane gases. Pairs of electrodes consisting of $\mathrm{Au} / \mathrm{Pd}(40 / 10 \mathrm{~nm})$ spaced by $400 \mathrm{~nm}$ are fabricated alongside the catalyst islands by standard electron-beam lithography techniques. Finally, bonding pads $(\mathrm{Au} / \mathrm{Cr} 150 / 10 \mathrm{~nm})$ are made by optical lithography and the devices are screened by room- and low-temperature measurements.

We measured the sample in an Oxford dilution refrigerator fitted with an Attocube ANRv51 piezo rotator, which enables high-precision in-plane rotation of the sample in large magnetic fields. The rotator provides resistive feedback of the actual position measured by lock-in techniques. For electrical filtering, room-temperature $\pi$-filters and low-temperature Thermocoax are used. The base temperature of the modified refrigerator is around $100 \mathrm{mK}$; all measurements are broadened by tunnel coupling, not temperature. The CNT measurement set-up consists of a National Instrument digital-to-analogue card, custom-made optically coupled amplifiers, a DL Instruments 1211 current-to-voltage amplifier and a Princeton Applied Research 5210 lock-in amplifier. Standard d.c. and lock-in techniques have been used to measure current and differential conductance $\mathrm{d} I / \mathrm{d} V_{\mathrm{sd}}$ whereas $\mathrm{d}^{2} I / \mathrm{d} V_{\mathrm{sd}}{ }^{2}$ is obtained numerically.

The bandgap of the device $2 \Delta_{g} \approx 30 \mathrm{meV}$ is measured directly as a large Coulomb diamond at $V_{g} \approx 1 \mathrm{~V}$, and in fitting the data of Fig. 4a to equation (1) we used $\varepsilon_{N} \approx 25 \mathrm{meV} / V \times V_{g}$ estimated from the level spacing $\Delta E \approx 3 \mathrm{meV}$ and the average Coulomb peak spacing $\left\langle\Delta V_{g}\right\rangle \approx 30 \mathrm{mV}$.

\section{Received 4 August 2010; accepted 8 November 2010;} published online 23 January 2011

\section{References}

1. Bulaev, D., Trauzettel, B. \& Loss, D. Spin-orbit interaction and anomalous spin relaxation in carbon nanotube quantum dots. Phys. Rev. B 77, 235301 (2008).

2. Fischer, J. \& Loss, D. Dealing with decoherence. Science 324, 1277-1278 (2009).

3. Churchill, H. et al. Electron-nuclear interaction in ${ }^{13} \mathrm{C}$ nanotube double quantum dots. Nature Phys. 5, 321-326 (2009).

4. Flindt, C., Sørensen, A. \& Flensberg, K. Spin-orbit mediated control of spin qubits. Phys. Rev. Lett. 97, 240501 (2006).

5. Trif, M., Golovach, V. \& Loss, D. Spin-spin coupling in electrostatically coupled quantum dots. Phys. Rev. B 75, 085307 (2007).

6. Nowack, K., Koppens, F., Nazarov, Y. \& Vandersypen, L. Coherent control of a single electron spin with electric fields. Science 318, 1430-1433 (2007).

7. Pfund, A., Shorubalko, I., Ensslin, K. \& Leturcq, R. Suppression of spin relaxation in an InAs nanowire double quantum dot. Phys. Rev. Lett. 99, 036801 (2007).

8. Kuemmeth, F., Ilani, S., Ralph, D. \& McEuen, P. Coupling of spin and orbital motion of electrons in carbon nanotubes. Nature 452, 448-452 (2008).

9. Kuemmeth, F., Churchill, H., Herring, P. \& Marcus, C. Carbon nanotubes for coherent spintronics. Mater. Today 13, 18-26 (March 2010).

10. Ando, T. Spin-orbit interaction in carbon nanotubes. J. Phys. Soc. Jpn 69, 1757-1763 (2000)

11. Chico, L., Lopez-Sancho, M. \& Munoz, M. Spin splitting induced by spin-orbit interaction in chiral nanotubes. Phys. Rev. Lett. 93, 176402 (2004).

12. Huertas-Hernando, D., Guinea, F. \& Brataas, A. Spin-orbit coupling in curved graphene, fullerenes, nanotubes, and nanotube caps. Phys. Rev. B 74, 155426 (2006).

13. Jeong, J. \& Lee, H. Curvature-enhanced spin-orbit coupling in a carbon nanotube. Phys. Rev. B 80, 075409 (2009).

14. Izumida, W., Sato, K. \& Saito, R. Spin-orbit interaction in single wall carbon nanotubes: Symmetry adapted tight-binding calculation and effective model analysis. J. Phys. Soc. Jpn 78, 074707 (2009).

15. Fasth, C., Fuhrer, A., Samuelson, L., Golovach, V. \& Loss, D. Direct measurement of the spin-orbit interaction in a two-electron InAs nanowire quantum dot. Phys. Rev. Lett. 98, 266801 (2007).

16. Csonka, S. et al. Giant fluctuations and gate control of the $g$-factor in InAs nanowire quantum dots. Nano Lett. 8, 3932-3935 (2008).

17. Nilsson, H. et al. Giant, level-dependent $\mathrm{g}$ factors in InSb nanowire quantum dots. Nano Lett. 9, 3151-3156 (2009).

18. Flensberg, K. \& Marcus, C. Bends in nanotubes allow electric spin control and coupling. Phys. Rev. B 81, 195418 (2010).

19. Liang, W., Bockrath, M. \& Park, H. Shell filling and exchange coupling in metallic single-walled carbon nanotubes. Phys. Rev. Lett. 88, 126801 (2002).

20. Cobden, D. \& Nygård, J. Shell filling in closed single-wall carbon nanotube quantum dots. Phys. Rev. Lett. 89, 046803 (2002).

21. Jarillo-Herrero, P. et al. Electronic transport spectroscopy of carbon nanotubes in a magnetic field. Phys. Rev. Lett. 94, 156802 (2005).

22. Makarovski, A., An, L., Liu, J. \& Finkelstein, G. Persistent orbital degeneracy in carbon nanotubes. Phys. Rev. B 74, 155431 (2006).

23. Moriyama, S., Fuse, T., Suzuki, M., Aoyagi, Y. \& Ishibashi, K. Four-electron shell structures and an interacting two-electron system in carbon-nanotube quantum dots. Phys. Rev. Lett. 94, 186806 (2005).

24. Oreg, Y., Byczuk, K. \& Halperin, B. Spin configurations of a carbon nanotube in a nonuniform externalpotential. Phys. Rev. Lett. 85, 365-368 (2000). 
25. Minot, E., Yaish, Y., Sazonova, V. \& McEuen, P. Determination of electron orbital magnetic moments in carbon nanotubes. Nature 428, 536-539 (2004).

26. Jarillo-Herrero, P., Sapmaz, S., Dekker, C., Kouwenhoven, L. \& van der Zant, H Electron-hole symmetry in a semiconducting carbon nanotube quantum dot. Nature 429, 389-392 (2004).

27. De Franceschi, S. et al. Electron cotunneling in a semiconductor quantum dot. Phys. Rev. Lett. 86, 878-881 (2001).

28. Paaske, J. et al. Non-equilibrium singlet-triplet Kondo effect in carbon nanotubes. Nature Phys. 2, 460-464 (2006).

29. Hanson, R., Kouwenhoven, L., Petta, J., Tarucha, S. \& Vandersypen, L. Spins in few-electron quantum dots. Rev. Mod. Phys. 79, 1217-1265 (2007).

30. Nygård, J., Cobden, D. \& Lindelof, P. Kondo physics in carbon nanotubes. Nature 408, 342-346 (2000).

31. Galpin, M., Jayatilaka, F., Logan, D. \& Anders, F. Interplay between Kondo physics and spin-orbit coupling in carbon nanotube quantum dots. Phys. Rev. B 81, 075437 (2010)

32. Kane, C. \& Mele, E. Size, shape, and low energy electronic structure of carbon nanotubes. Phys. Rev. Lett. 78, 1932-1935 (1997).

33. Kleiner, A. \& Eggert, S. Band gaps of primary metallic carbon nanotubes. Phys. Rev. B 63, 073408 (2001).

34. Chico, L., Lopez-Sancho, M. \& Munoz, M. Curvature-induced anisotropic spin-orbit splitting in carbon nanotubes. Phys. Rev. B 79, 235423 (2009).
35. Kong, J., Soh, H., Cassell, A., Quate, C. \& Dai, H. Synthesis of individual single-walled carbon nanotubes on patterned silicon wafers. Nature 395, 878-881 (1998).

\section{Acknowledgements}

We thank P. E. Lindelof, J. Mygind, H. I. Jørgensen, C. M. Marcus and F. Kuemmeth for discussions and experimental support. T.S.J. acknowledges the Carlsberg Foundation and Lundbeck Foundation for financial support. K.G-R., K.F. and J.N. acknowledge The Danish Research Council and University of Copenhagen Center of Excellence.

\section{Author contributions}

T.S.J. and K.G-R. made the measurements, analysed the data and wrote the paper. T.S.J. designed the rotating sample stage. K.G-R. made the sample. K.M., T.F. and J.N participated in discussions and writing the paper. J.P. and K.F. developed the theory and guided the experiment.

\section{Additional information}

The authors declare no competing financial interests. Supplementary information accompanies this paper on www.nature.com/naturephysics. Reprints and permissions information is available online at http://npg.nature.com/reprintsandpermissions. Correspondence and requests for materials should be addressed to T.S.J. or K.G-R. 